OPEN ACCESS

Edited by:

Jean-Pierre Sao-Ming Lin,

Guy's and St Thomas' NHS

Foundation Trust, United Kingdom

Reviewed by:

Angelo Lavano,

University of Magna Graecia, Italy

Alice Bonuccelli,

Pisana University Hospital, Italy

*Correspondence:

Diane Ruge

diane.ruge@gmail.com

Specialty section:

This article was submitted to

Pediatric Neurology,

a section of the journal

Frontiers in Neurology

Received: 06 August 2021

Accepted: 18 October 2021

Published: 30 November 2021

Citation:

Trenado C, Cif L, Pedroarena-Leal N and Ruge D (2021)

Electrophysiological Signature and the

Prediction of Deep Brain Stimulation Withdrawal and Insertion Effects.

Front. Neurol. 12:754701.

doi: 10.3389/fneur.2021.754701

\section{Electrophysiological Signature and the Prediction of Deep Brain Stimulation Withdrawal and Insertion Effects}

\author{
Carlos Trenado ${ }^{1}$, Laura Cif ${ }^{2}$, Nicole Pedroarena-Leal ${ }^{1}$ and Diane Ruge ${ }^{1 *}$ \\ 1 Laboratoire de Recherche en Neurosciences Cliniques, LRENC, Montpellier, France, ' 2 Département de Neurochirurgie, \\ Centre Hospitalier Universitaire de Montpellier, Montpellier, France
}

Deep brain stimulation (DBS) serves as a treatment for neurological and psychiatric disorders, such as Parkinson's disease (PD), essential tremor, dystonia, Tourette Syndrome (GTS), Huntington's disease, and obsessive-compulsive disorder (OCD). There is broad experience with the short-term effects of DBS in individual diseases and their signs/symptoms. However, even in acute treatment and for the same disorder or a given disorder, a prediction of effect is not perfect. Even further, the factors that influence the long-term effect of DBS and its withdrawal are hardly characterized. In this work, we aim to shed light on an important topic, the question of "DBS dependency." To address this, we make use of the Kuramoto model of phase synchronization (oscillation feature) endowed with neuroplasticity to study the effects of DBS under successive withdrawals and renewals of neuromodulation as well as influence of treatment duration in de novo DBS "patients." The results of our simulation show that the characteristics of neuroplasticity have a profound effect on the stability and mutability of oscillation synchronization patterns across successive withdrawal and renewal of DBS in chronic "patients" and also in de novo DBS "patients" with varying duration of treatment (here referred to as the "number of iterations"). Importantly, the results demonstrate the strong effect of the individual neuroplasticity makeup on the behavior of synchrony of oscillatory activity that promotes certain disorder/disease states or symptoms. The effect of DBS-mediated neuromodulation and withdrawal is highly dependent on the makeup of the neuroplastic signature of a disorder or an individual.

Keywords: electrophysiological signature, deep brain stimulation, dependency, neuropsychiatric disease, neuromodulation

\section{INTRODUCTION}

Deep brain stimulation (DBS) is a neuromodulation technique that is effective as a treatment for severe neurological and psychiatric disorders (1-4). It has been suggested that it modulates cortico-striatal brain circuitry with an indirect effect on cognitive and behavioral abilities $(5,6)$. The overall efficacy of DBS for different pathologies, such as Parkinson's disease (PD) and dystonia, has been well-established for months and a number of years. The long-term effects remain overall effective on motor symptoms and mood $(7,8)$; they have, however, to be characterized further 
in terms of variability in efficacy and clinical adverse features, such as stimulation-related side effects or stimulation-independent effects. To further the demographic and history of disease-related predictors, understanding of relevant prediction factors for short- and long-term effects of DBS is required. Long-term neuromodulation with deep brain stimulation reorganizes the brain, changes the inherent patterns of cortical excitability typical of a particular disorder or symptom, and causes different individual clinical responses of a patient upon withdrawal of the stimulation input. One meaningful marker for the clinical withdrawal effect seems to be neuroplasticity which is quantifiable with electrophysiological recordings (9-12). Importantly, in patients in vivo it would be impossible to characterize the complex multifactorial patterns of neuroplasticity in a specific state (e.g., "ON DBS," "OFF DBS", and "symptom status") the patient is in. The reasons for this are technical in nature. This explains the high value of a computational simulation as used here. It allows consideration of an input as a function of complex patterns, supposedly reflecting an electrophysiological signature of a patient.

A mechanism by which complex systems reach a specific state is synchronization of the lower-level elements that are organized into a functional unit (13). Synchronization has been referred to as the property of a non-linear system in which the dynamics of individual elements are correlated in time (14). Computational models have shown that synchronized spiking within small neural populations in cortical and hippocampal areas may be enhanced through Hebbian learning, which is characterized by long-term potentiation (LTP) if a presynaptic spike precedes a postsynaptic spike within a brief time window or by long-term depression (LTD) if the temporal order of spikes is reversed, a relationship described as neurons that fire together wire together (15-18). The invasive and non-invasive brain stimulation approaches allow a quantification of synaptic strength in the human nervous system, and manipulation of it has implications for the treatment of neurological and psychiatric disorders (19, 20). Exaggerated oscillatory neuronal synchronization relates to the cardinal symptoms of bradykinesia, rigidity, dystonia, and levodopa-induced dyskinesias. Excessive theta synchronization is a finding in dystonia, sensorimotor integration, and motor learning (21-25). Excessive beta oscillations have often been linked to specific Parkinson symptoms. It has been hypothesized that DBS is able to interrupt pathological synchronization (26).

At large-scale levels, for instance by considering electroencephalography (EEG) and magnetoencephalography (MEG) data, a valid index of synchronization is in-phase activation of neural elements in relation to cognition and pathology. In particular, alpha-beta phase synchronization has been reported to mediate the recruitment of visuospatial attention (27), while the role of selective attention in controlling phase oscillatory neural activity to efficiently process relevant information at pre-stimulus stages has been emphasized (28). In addition, frontotemporal theta phase-synchronization has been shown to underlie music-evoked pleasantness (29), while inter-brain phase synchronization has been proposed to be a marker of human social interaction (30). With regards to pathology, previous studies reported that deficits in EEG phase synchrony may underlie cognitive disturbances in schizophrenia (31) and that aberrant multi-frequency MEG phase temporal synchronization may be useful to predict conversion from mild cognitive impairment to Alzheimer's disease (AD) (32). Likewise, EEG phase synchrony has been helpful to prognosticate the outcomes in pediatric coma (33). Mean phase coupling of the motor brain regions has been shown to be abnormally enhanced in patients with $\mathrm{PD}$ and isolated dystonia $(34,35)$. In contrast, pianists with musician's dystonia exhibited deficient phase coupling between the neuronal assemblies required to inhibit motor memory traces (36). Increased EEG phase synchronization in all bands has been shown to be present in patients with Huntington's disease and to correlate with cognitive decline (37). The enhanced coupling or synchronization seems to be a feature of pathology, e.g., rigidity in PD or dystonic symptoms in dystonia. The desynchronization (or decoupling) on the other hand rather reflects "leaving of (also pathological) state," in line with Pfurtscheller's work (38), while synchronization in the oscillations has a physiological healthy function (e.g., idling states) and the lack of mutability (change between states) bears pathology. For clinicians who use DBS as a treatment, pressing questions are: "What happens with the patient when I start DBS, when I switch off DBS after a short treatment duration, after a long treatment duration, when it accidentally stops working"? And moreover, "Is the patient dependent on the DBS or is there a window in time where I can ultimately stop DBS and the patient reaches independence?"

In the present study, we made use of computational modeling using an established network's model of synchronization, Kuramoto's model, endowed with plasticity (39). First, we targeted the effect of consecutive withdrawals and renewals of DBS by considering the different neuroplasticity conditions defined by the levels of (long term) potentiation and depotentiation. Second, we examined the effect of stimulation duration (by varying the number of iterations in the model) in de novo DBS "patients," again under different neuroplasticity conditions. The results of our computer simulation mirror relevant clinical observations and also broaden our understanding of the long-term effect of DBS under the considered conditions.

\section{MATERIALS AND METHODS}

\section{Kuramoto's Model With Endowed Plasticity}

As emphasized by previous DBS studies, the state of a neuron can be described by a set of variables that for certain parameters display a regular behavior. Therefore, such a state is susceptible to be described by the parameters that reflect changes in regularity as in the case of the phase (40). The same notion naturally applies to the ensembles of neurons whose regular behavior gives place to the patterns of regularity that have been linked to high cognitive functions and behavioral features as described earlier. On the basis of such an observation, we adopt Kuramoto's model of network synchronization to address the long-term effects of DBS. 
In accordance with Kuramoto's model, the phase evolution equation for a network of coupled oscillators is given by:

$$
\frac{\partial}{\partial t} \varphi_{i}=\omega_{i}+\frac{K_{i j}}{N} \sum_{i=1}^{N} \sin \left(\varphi_{i}-\varphi_{j}\right)+I_{i}
$$

where $\varphi_{i}$ and $\omega_{i}$ represent the phase and natural frequency of oscillator $i, K_{i j}$ refers to the coupling between the oscillators $i$ and $j, N$ represents the number of oscillators $(N=100)$, and $I_{i}$ denotes the DBS input received by the oscillator $i$. The values for $\omega_{i}$ were uniformly distributed random numbers in the interval $(0,1)$. The DBS input adopted was a stereotypical train of rectangular pulses with a $130 \mathrm{~Hz}$ frequency and a 3.0 amplitude. Note that a modified version of this model has been previously used to evaluate the efficacy of new therapeutic DBS protocols (40). As in previous studies (39), we assume a direct effect of plasticity on the coupling between the oscillators as defined by:

$$
K_{i j}=a_{p} \star \exp \left(\frac{r_{1}}{\tau_{p}}\right)-\alpha_{d} \exp \left(\frac{r_{2}}{\tau_{d}}\right),
$$

where $\alpha_{p}$ and $\alpha_{d}$ refer to the potentiation and depotentiation rates, $\tau_{p}$ and $\tau_{d}$ denote the damping parameters (set-up as 0.5 ), and $r_{1}$ and $r_{2}\left(r_{1} \neq r_{2}\right)$ denote constant parameters that were selected from the uniformly distributed random values in the interval $(0,1)$ for each pair of oscillators $i, j$.

\section{Synchronization Quantification}

To quantify global synchronization for the considered network of coupled oscillators, we make use of the phase locking value (PLV) (41), which provides a normalized synchronization index [ranging from 0 (no synchronization) to 1 (full synchronization)] between a pair of oscillators $i, j=1.0 .100$ as defined by:

$$
P L V_{i j}=\frac{1}{N}\left|\sum_{i=1}^{N} e^{-i\left(\varphi_{i}-\varphi_{j}\right)}\right|
$$

where $\varphi_{i}$ and $\varphi_{j}$ denote the phase of oscillators $i$ and $j$. The grand average of $P L V_{i j}$ across all possible combinations of pairs of oscillators ( $i \neq j$ and without repetition) represents a global index of network synchronization for a given plasticity and DBS condition.

\section{Plasticity Conditions}

In the present study, we considered different plasticity conditions on the basis of previous studies addressing the assessment of neuroplasticity in the case of patients suffering from neurodegenerative and psychiatric disorders as well as healthy subjects. For instance, a reduction of neuroplasticity as reflected in decreased LTP and LTD has been suggested in subjects with depression by transcranial magnetic stimulation (TMS) studies utilizing paired associative stimulation (PAS) (42). A deficient plasticity that is reflected in strong asymmetry of LTP and LTD has been suggested in patients suffering from bipolar disorder by studies targeting the effect of lithium on human plasticity (43). With regards to PD, lack of LTP in the primary motor cortex has been stressed by TMS studies utilizing intermittent theta burst stimulation (iTBS) (44). An excess of LTP has been suggested in the case of patients with dystonia (45).

Note that the plasticity conditions in the adopted synchronization model are defined by setting up the specific values for the potentiation and depotentiation rate parameters; specifically the following plasticity conditions were considered:

(1) high level of potentiation $\left(\alpha_{p}=8.0\right)$ and low level of depotentiation $\left(\alpha_{d}=0.001\right)$; (2) low level of potentiation $\left(\alpha_{p}=\right.$ $0.001)$ and high level of depotentiation $\left(\alpha_{d}=8.0\right)$; (3) equally high level of potentiation $\left(\alpha_{p}=8.0\right)$ and depotentiation $\left(\alpha_{d}=\right.$ 8.0); (4) equally medium level of potentiation $\left(\alpha_{p}=4.0\right)$ and depotentiation $\left(\alpha_{d}=4.0\right)$; equally low level of potentiation and depotentiation: (5) $\left(\alpha_{p}=0.7\right)$ and $\left(\alpha_{d}=0.7\right)$; (6) $\left(\alpha_{p}=0.1\right)$ and $\left(\alpha_{d}=0.1\right)$; and (7) $\left(\alpha_{p}=0.001\right)$ and $\left(\alpha_{d}=0.001\right)$.

\section{DBS Conditions}

Focusing on the effect of consecutive withdrawal and renewal of DBS, the conditions DBS ON, DBS OFF, DBS ON2, DBS OFF2, and DBS ON3 were considered. For these conditions, duration and absence of DBS were set up to 2,000 iterations.

In the case of de novo DBS "patients," the conditions DBS OFF and DBS ON were considered. For these conditions, duration, and absence of DBS stimulation were set up to 500, 1,000, and 2,000 iterations.

\section{Synchronization Percentage Change}

For the long-term scenario of consecutive withdrawal and renewal of DBS, the condition DBS ON was defined as the baseline level (100\%) so that percentage change in synchronization for the subsequent DBS conditions (DBS OFF, DBS ON2, DBS OFF2, and DBS ON3) was calculated in relation to DBS ON. Analogously, the initial condition DBS OFF was adopted as the baseline level (100\%) in the case of de novo DBS “patients.”

\section{RESULTS}

With a focus on the long-term effect of DBS under successive withdrawal and renewal of stimulation, we varied the level and balance of potentiation and depotentiation as depicted in Figures 1A,B, 2A,B. Strikingly, a stable high phase locking value was noticeable in the case of high potentiation and low depotentiation (Figures 1A, 2A), whereas PLV fluctuated between increased and decreased very low values. There is a stable increase of PLV during the successive DBS OFF states in the opposite constellation of potentiation and depotentiation (Figures 1B, 2B). The case of symmetry (i.e., equally high, medium, or low) in the level of potentiation and depotentiation is depicted by Figures $\mathbf{1 C}-\mathbf{G}, \mathbf{2 C}-\mathbf{G}$. It is noticeable that for the high levels of potentiation and depotentiation (8.0 and 8.0), PLV tended to decrease under successive DBS withdrawal and renewals (Figures 1C, 2C); in the case of middle levels of potentiation and depotentiation (4.0 and 4.0), PLV showed a stable trend without fluctuations (Figures 1D, 2D); in the case of low values of potentiation and depotentiation (0.7 and 0.7), PLV fluctuated between increased and decreased low values with a consistent increase during the successive DBS OFF states (Figures 1E, 2E). In the case of very low levels of potentiation 


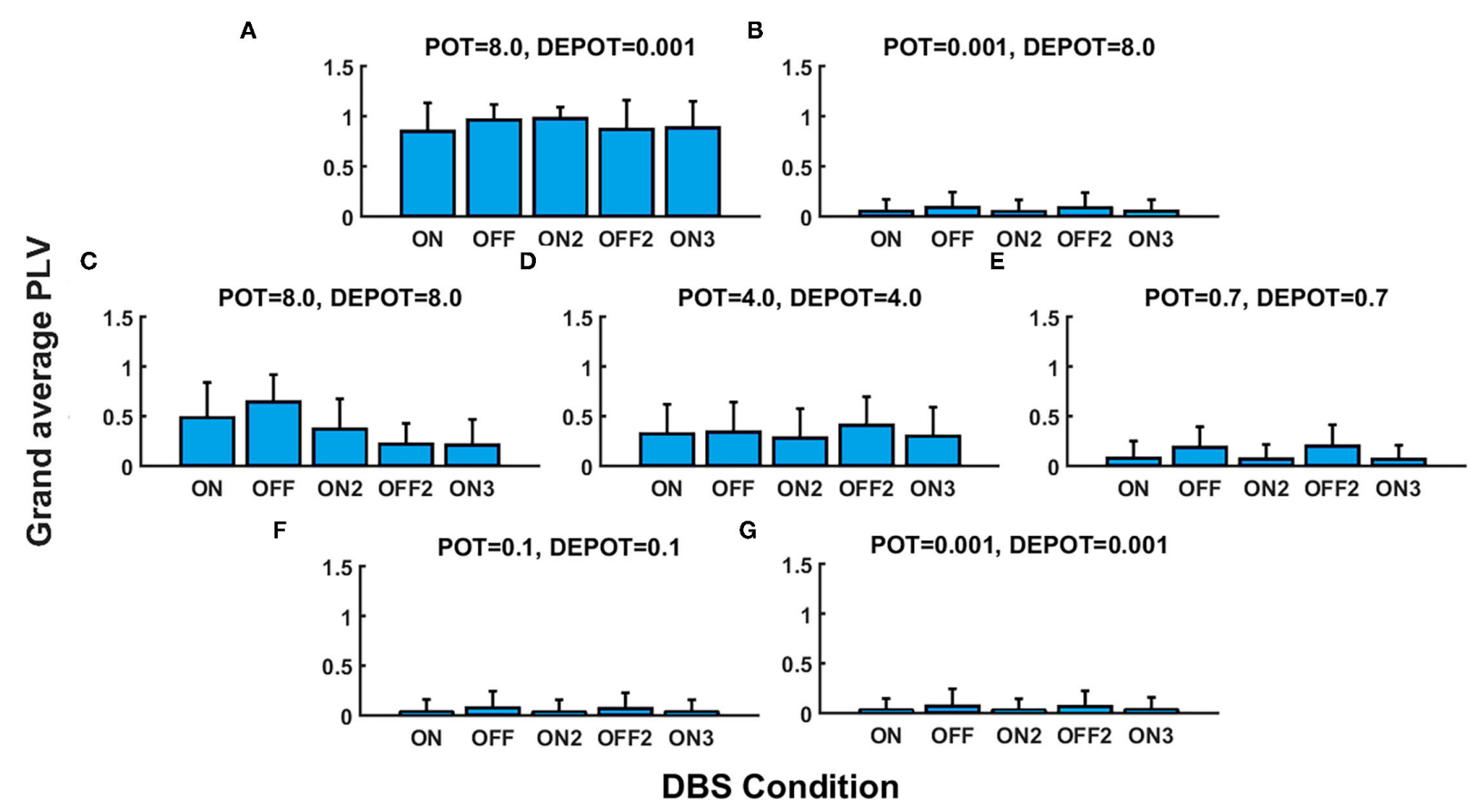

FIGURE 1 | The simulation results (mean and SD of the grand average phase locking value [PLV]) corresponding to the long-term effect of deep brain stimulation (DBS) under successive withdrawal and renewal of stimulation: unbalanced potentiation and depotentiation (high and low level) (A,B); balance potentiation and depotentiation, i.e., equally high levels, (C); equally medium levels (D); equally low levels (E-G).

and depotentiation (0.1 as well as 0.001$)$, PLV fluctuated between increased and decreased very low values with a stable increase during the successive DBS OFF states (Figures 1F,G, 2F,G).

With a focus on longitudinal development under DBS input in de novo DBS “patients," we again altered the level and balance of potentiation and depotentiation as depicted by Figures 3A,B, 4A,B. Strikingly, a stable high level of PLV was noticeable across a number of iterations (500, 1,000, and 2,000) (Figure 3A) and thus reflected the duration of DBS input, with a slight increase during DBS ON (Figure 4A) in the case of high potentiation and low depotentiation. A stable low level of PLV was noticeable across a number of iterations (500, 1,000, and 2,000) (Figure 3B) with a tendency to decrease during DBS OFF and DBS ON (Figure 4B) in the case of low potentiation and high depotentiation. The case of symmetry in the level of potentiation and depotentiation is depicted in Figures 3C-E, 4C-E. It is noticeable that for high levels of potentiation and depotentiation (8.0 and 8.0), PLV first increased and then showed a tendency to decrease in the transition from DBS OFF to DBS ON across a number of iterations (Figures 3C, 4C); in the case of middle values of potentiation and depotentiation (4.0 and 4.0), PLV first increased and then showed a tendency to decrease during DBS OFF while a tendency to decrease was observed during DBS ON across a number of iterations (Figures 3D, 4D); in the case of very low levels of potentiation and depotentiation (0.001 and 0.001), PLV showed low values with a tendency to decrease during DBS OFF and DBS ON across a number of iterations (Figures 3E, 4E).

\section{DISCUSSION}

This paper summarizes a computational modeling study in scenarios with different neuroplasticity distributions, reflecting virtual "patients" with different neurophysiological signatures. When a powerful treatment, such as deep brain stimulation gets introduced in a new patient, questions arise as to "Why there is no effect? Will there be an effect when I stimulate for a longer time? What happens when the DBS machinery fails or a planned interruption of the stimulation occurs?" In long-term patients on this neuromodulation treatment, the question comes up whether a break or time off the intervention could be planned, or whether a life-long dependence on this input is likely.

In line with existing neurobiological models and neurophysiological findings in various DBS-treated conditions, our study looks at synchronization (coupling) of oscillatory activity, assuming that too much synchronization in certain frequency bands (beta in $\mathrm{PD}$, theta in dystonia, etc.) maintains the pathological state (rigidity, dystonic symptoms, obsessive compulsive behavior, etc.), whereas a reduction in synchronization reflects the leaving of the symptom-stabilizing state. The in vivo situation of a biological system of course is complex and the exact mechanism of action leading to symptom improvement initiated by disruption of the synchronization patterns through DBS remains unclear (46).

The adopted computational simulation approach is particularly advantageous with regards to avoiding the risks 


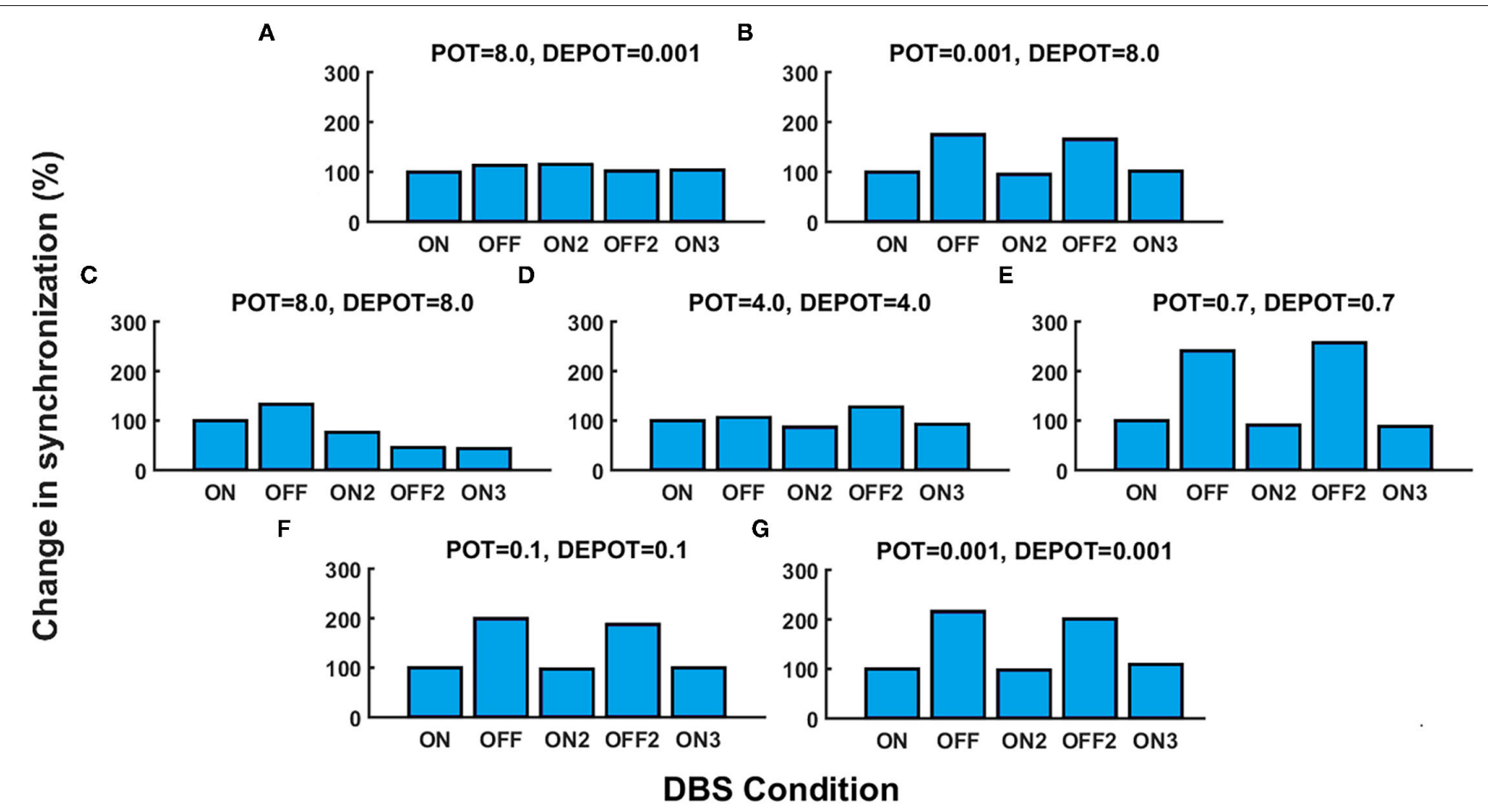

FIGURE 2 | The simulation results [percentage change of the grand average PLV, DBS ON represents the baseline level (100\%)] corresponding to the long-term effect of DBS under successive withdrawal and renewal of stimulation: unbalanced potentiation and depotentiation (high and low level) (A,B); balance potentiation and depotentiation, i.e., equally high levels, (C); equally medium levels (D); equally low levels (E-G).

associated with turning on and off an implanted DBS device in patients suffering from neurological or psychiatric pathology and allowing flexibility in setting up the different plasticity scenarios that would not be accessible simultaneously under real (in vivo) patient conditions.

In the first part of our study, we simulated patients with DBS switched on long-term, and observed the effect of consecutive withdrawals and renewals of DBS. In the second part of the study, de novo "patients" (with no previous DBS treatment) received long-term DBS of various durations. On DBS treatment, the literature shows that certain symptoms seem to respond very quickly (tremor in PD) whereas others need longer, yet variable, time to allow the DBS effects on symptom alleviation to occur (dystonia) (9, 47). Particular patient profiles, that is, individual differences in baseline potentiation or depotentiation changes due to symptoms, have never been profiled and could provide information as to the potential response patients have to neuromodulation settings and treatment duration.

Our study introduces different neuroplasticity makeups, i.e., different levels and balances of potentiation and depotentiation. It assumes that this mirrors some real patient electrophysiological signatures. It is well-established that dystonia tends to show too much neuroplasticity, whereas it tends to be at normal levels in obsessive-compulsive disorders (OCD), and there is a lack of it in $\mathrm{PD}$, Tourette Syndrome, bipolar disorder, and schizophrenia, as examples for abnormalities (48-50). These citations all represent group level studies, however, individual neuroplasticity levels are relevant for the personal effect of withdrawal or insertion of therapeutic input.

For the first part of the study, the simulations revealed that symmetry and asymmetry in the potentiation and depotentiation levels have a strong effect on the stability and level of synchronization patterns across the successive withdrawals and renewals of DBS input to the system. Our results reveal that, interestingly, a high level of potentiation in combination with a low level of depotentiation ensures the system is "stuck" in its current state (low mutability). Whether DBS is switched on or off does not affect the oscillatory state of the system. It remains stabilized at its observable high level of synchronization. On the contrary, when potentiation is low, independent of whether this is in combination with high or low depotentiation values, the oscillatory state of the system is highly mutable with a striking effect of switching DBS on or off. However, the effects of switching DBS on or off seem highly predictable. Intriguingly, when potentiation and depotentiation are balanced, both high or medium, the response to repeated insertions and withdrawals of DBS becomes less predictable and might suggest that they both counteract each other in line with a homeostatic regulation of the system, where a drive in one direction is counteracted via the other mechanism and driven into the opposite direction, with the goal of keeping the system in healthy boundaries. We conclude that the reaction of the oscillatory system highly depends on 




the neuroplasticity makeup of the "individual." Let us assume that there are disorders with too much plasticity, i.e., too much potentiation: In this case, the reaction of the system to DBS withdrawal or reinsertion is almost ineffective. The system is stabilized in its current status. This situation resembles that of many DBS naïve dystonia patients, for example. The long time to respond to initial treatment in dystonia or the strong resistance to neuromodulation and to occupational training might be due to such a neurophysiological signature. In the low potentiation constellation, the oscillatory system instantly responds to being switched on or off. The system is highly mutable, a response well-known for major symptoms of PD and long-term DBS-treated dystonia patients who have low potentiation as a cardinal neurophysiological feature $(9,47)$.
The change of the oscillatory system directly matches the ON/OFF state and is highly predictable. The most complicated constellation arises with balanced high or medium levels of potentiation and depotentiation. Unlike what happens in the high unbalanced potentiation situation, in this case, the system responds to insertion and withdrawal of DBS. However, the contradictory forces of neuroplasticity seem to intermingle and one might speculate that the homeostatic mechanisms come into play to keep the system within healthy boundaries. Here, the outcome of DBS OFF and ON scenarios becomes unpredictable. However, change happens, and this stands in contrast to the unbalanced high potentiation-low depotentiation scenario where the oscillatory system remains immutable and "stuck" in its current state. 


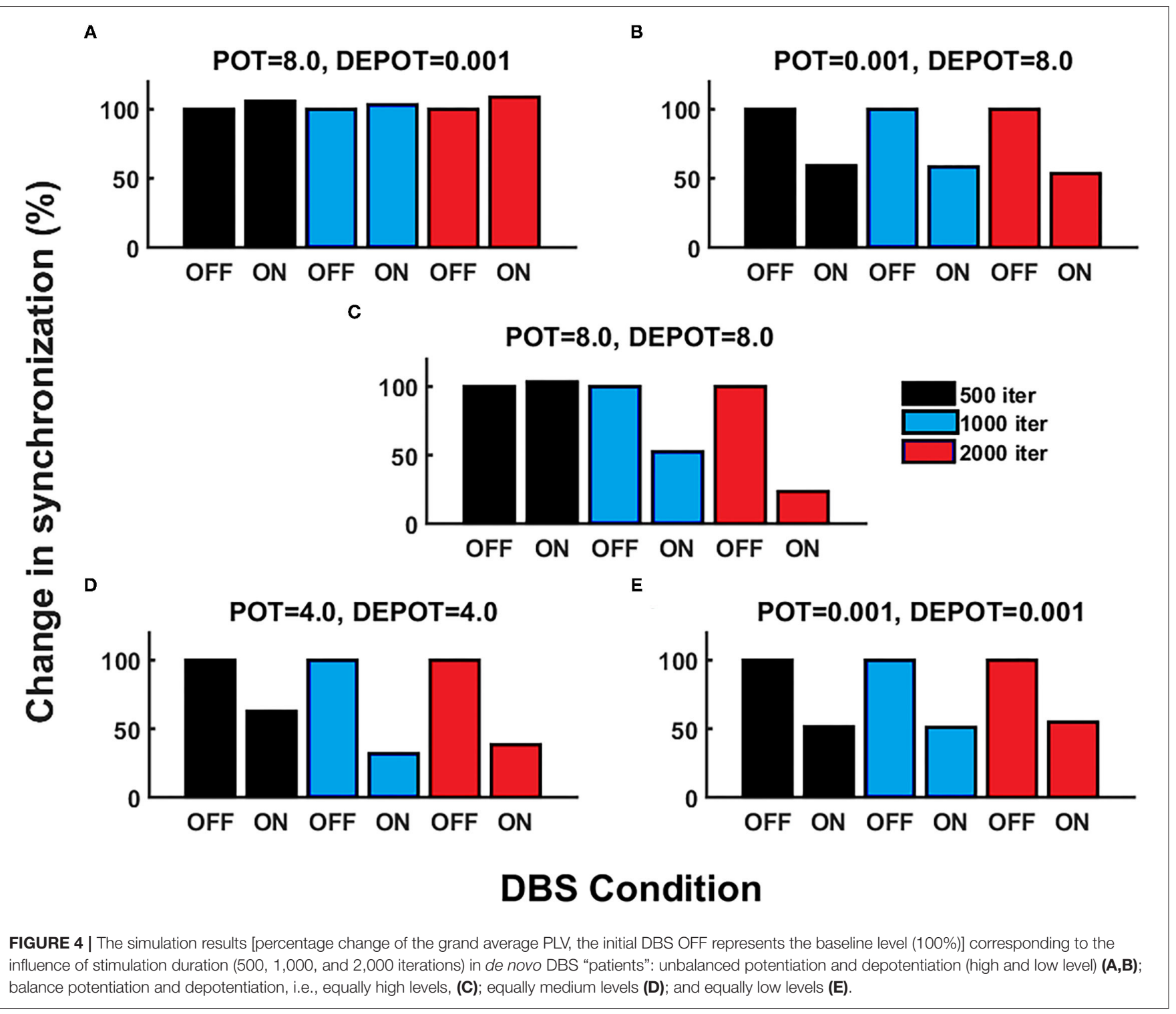

In the second part of our study, the computational simulation mimicked a de novo "patient" who then received DBS for various durations (iterations), and consecutively withdrawal of DBS was simulated at different time points along this time axis. Again, as in the first part of the study, the outcome was highly dependent on the synaptic plasticity signature. In the unbalanced high potentiation-low depotentiation situation, the system over time remains immutable and "stuck" in its oscillatory state. DBS insertion is not able to produce change in the system, resembling the situation of therapy-resistant patients. As an example, naïve patients with dystonia are known for their high potentiation. The removal of high potentiation, allowing the system to become mutable, might drive the change toward the beneficial effects of DBS as suggested before (9). In the balanced high and medium potentiation-depotentiation scenario, we observe effects in a monophasic positive direction after insertion of DBS with a gradual decrease in coupling in the oscillatory system. Interestingly, however, upon withdrawal of DBS, a rebound occurs that exceeds the level of the initial coupling by far. This might resemble a situation of dramatic, sometimes life threatening worsening of symptoms in dystonia patients with accidental or planned DBS switch OFF (51). In a scenario where there is an unbalanced or balanced low potentiation, the response to DBS is present and in a positive direction, it appears that the system is mutable. Intriguingly, the oscillatory system reverts back to the DBS naive state upon DBS withdrawal, but not to the $100 \%$ baseline level of the naïve system, in other words some of the DBSinduced effect seems to be stabilized despite a relative lack of potentiation. However, this is only a mild deviation from the naïve $100 \%$ value in the OFF state (before treatment or DBS input was initiated).

The computational simulation study shows the strong effect of the individual neuroplasticity makeup on the insertion and 
withdrawal of DBS as a therapeutic tool on the oscillatory system and thereby supposedly on disorder symptoms manifesting in brain coupling. How mutable a system is and thereby how effectively it responds to the treatment input might be linked to such individual signatures. Even in healthy people, neuroplasticity levels are variable and individual $(52,53)$. The meaning of such a marker setup or signature for personalized therapy and management of patients becomes clear by the usage of such computational approaches.

Limitations of the study or points to consider: This is a computational modeling and simulation approach and not in vivo data. However, in this limitation lies strength, because it is impossible, due to technical limitations, to obtain such data during acquisition of this type of neurophysiological recordings in real patients. Besides technical impossibility, the potential harm of switch OFF situations needs to be carefully considered by experienced clinicians who know the patients well. The second point to consider is that this modeling does not currently include the fact that the neuroplasticity itself is dependent on the oscillatory system and therefore will be dynamic over time. In other words, the results of the current study reflect the neuroplasticity conditions that once set up remain the same across iterations. As shown previously, the neuroplasticity is

\section{REFERENCES}

1. Vijverman AC, Fox SH. New treatments for the motor symptoms of Parkinson's disease. Expert Rev Clin Pharmacol. (2014) 7:761-77. doi: 10.1586/17512433.2014.966812

2. Groiss SJ, Wojtecki L, Südmeyer M, Schnitzler A. Deep brain stimulation in Parkinson's disease. Ther Adv Neurol Disord. (2009) 2:20-8. doi: 10.1177/1756285609339382

3. Lee DJ, Lozano CS, Dallapiazza RF, Lozano AM. Current and future directions of deep brain stimulation for neurological and psychiatric disorders. $J$ Neurosurg. (2019) 131:333-42. doi: 10.3171/2019.4.JNS181761

4. Harmsen IE, Elias GJB, Beyn ME, Boutet A, Pancholi A, Germann J, et al. Clinical trials for deep brain stimulation: current state of affairs. Brain Stimul. (2020) 13:378-85. doi: 10.1016/j.brs.2019.11.008

5. Calabresi P, Ghiglieri V, Mazzocchetti P, Corbelli I, Picconi B. Levodopainduced plasticity: a double-edged sword in Parkinson's disease? Philos Trans $R$ Soc Lond B Biol Sci. (2015) 370:20140184. doi: 10.1098/rstb.2014.0184

6. Da Cunha C, Boschen SL, Gómez,-A. A., Ross EK, Gibson WSJ, Min $\mathrm{HK}$, et al. Toward sophisticated basal ganglia neuromodulation: review on basal ganglia deep brain stimulation. Neurosci Biobehav Rev. (2015) 58:18610. doi: 10.1016/j.neubiorev.2015.02.003

7. Cif L, Demailly D, Lin JP, Barwick KE, Sa M, Abela L, et al. KMT2B-related disorders: expansion of the phenotypic spectrum and long-term efficacy of deep brain stimulation. Brain. (2020) 143:324261. doi: 10.1093/brain/awaa304

8. Krause P, Völzmann S, Ewert S, Kupsch A, Schneider GH, Kühn AA. Long-term effects of bilateral pallidal deep brain stimulation in dystonia: a follow-up between 8 and 16 years. J Neurol. (2020) 267:162231. doi: 10.1007/s00415-020-09745-Z

9. Ruge D, Tisch S, Hariz MI, Zrinzo L, Bhatia KP, Quinn NP, et al. Deep brain stimulation effects in dystonia: time course of electrophysiological changes in early treatment. Mov Disord. (2011) 26:1913-21. doi: 10.1002/mds.2 3731

10. Ruge D, Cif L, Limousin P, Gonzalez V, Vasques X, Hariz MI, et al. Shaping reversibility? Long-term deep brain stimulation in dystonia: the relationship between effects on electrophysiology and clinical symptoms. Brain. (2011) 134:2106-15. doi: 10.1093/brain/awr122 variable over time and also influences the switch OFF clinical outcome based on its potentiation as one form of neuroplasticity at that particular time point $(9,11,12,54)$. To explore the dynamics of the system would be a meaningful next study.

In conclusion, the electrophysiological signature has a profound impact on the effects of an intervention, such as DBS on the system, and can be used in future to narrow down potential outcomes in specific scenarios.

\section{DATA AVAILABILITY STATEMENT}

The raw data supporting the conclusions of this article will be made available by the authors, without undue reservation.

\section{AUTHOR CONTRIBUTIONS}

All authors listed have made a substantial, direct, and intellectual contribution to the work and approved it for publication.

\section{ACKNOWLEDGMENTS}

We acknowledge the Dorothy Feiss Scientific Research Grant.

11. Ruge D, Cif L, Limousin P, Gonzalez V, Vasques X, Coubes $\mathrm{P}$, et al. Longterm deep brain stimulation withdrawal: clinical stability despite electrophysiological instability. J Neurol Sci. (2014) 342:197-9. doi: 10.1016/j.jns.2014.05.011

12. Cif L, Ruge D, Gonzalez V, Limousin P, Vasques X, Hariz MI, et al. The influence of deep brain stimulation intensity and duration on symptoms evolution in an OFF stimulation dystonia study. Brain Stimul. (2013) 6:5005. doi: 10.1016/j.brs.2012.09.005

13. Nowak A, Vallacher RR, Zochowski M, Rychwalska A. Functional synchronization: the emergence of coordinated activity in human systems. Front Psychol. (2017) 8:945. doi: 10.3389/fpsyg.2017.00945

14. Pikovsky A, Rosenblum MG, Kurths J. Synchronization: a universal concept in nonlinear sciences. Am J Phys. (2002) 70:655. doi: 10.1119/1.1475332

15. Suri RE, Sejnowski TJ. Spike propagation synchronized by temporally asymmetric Hebbian learning. Biol Cybern. (2002) 87:440-5. doi: 10.1007/s00422-002-0355-9

16. Debanne D, Gahwiler BH, Thompson SM. Long-term synaptic plasticity between pairs of individual CA3 pyramidal cells in rat hippocampal slice cultures. J Physiol. (1998) 507:237-47. doi: 10.1111/j.1469-7793.1998.237bu.x

17. Feldman DE. Timing-based LTP and LTD at vertical inputs to layer II/III pyramidal cells in rat barrel cortex. Neuron. (2000) 27:45-56. doi: 10.1016/S0896-6273(00)00008-8

18. Hebb DO. The Organization of Behaviour. A Neuropsychological Theory. John Wiley and Sons (1949).

19. Herrington TM, Cheng JJ, Eskandar EN. Mechanisms of deep brain stimulation. J Neurophysiol. (2016) 115:19-38. doi: 10.1152/jn.00281.2015

20. Terranova C, Rizzo V, Cacciola A, Chillemi G, Calamuneri A, Milardi D, et al. Is there a future for non-invasive brain stimulation as a therapeutic tool? Front Neurol. (2019) 24:1146. doi: 10.3389/fneur.2018.01146

21. Chen CC, Kuhn AA, Trottenberg T, Kupsch A, Schneider GH, Brown P. Neuronal activity in globus pallidus interna can be synchronized to local field potential activity over $3-12 \mathrm{~Hz}$ in patients with dystonia. Exp Neurol. (2006) 202:480-6. doi: 10.1016/j.expneurol.2006.07.011

22. Bland $\mathrm{BH}$, Oddie SD. Theta band oscillation and synchrony in the hippocampal formation and associated structures: the case for its role in sensorimotor integration. Behav Brain Res. (2001) 127:119-36. doi: 10.1016/S0166-4328(01)00358-8 
23. DeCoteau WE, Thorn C, Gibson DJ, Courtemanche R, Mitra P, Kubota Y, et al. Learning-related coordination of striatal and hippocampal theta rhythms during acquisition of a procedural maze task. Proc Natl Acad Sci USA. (2007) 104:5644-9. doi: 10.1073/pnas.0700818104

24. Gengler S, Mallot HA, Holscher C. Inactivation of the rat dorsal striatum impairs performance in spatial tasks and alters hippocampal theta in the freely moving rat. Behav Brain Res. (2005) 164:73-82. doi: 10.1016/j.bbr.2005.06.009

25. Trenado C, Hartmann CJ, Elben S, Pauls KAM, Friggemann L, Groiss SJ, et al. Local field potential oscillations of the globus pallidus in cervical and tardive dystonia. J Neurol Sci. (2016) 366:68-73. doi: 10.1016/j.jns.2016.04.033

26. Brown P. Abnormal oscillatory synchronisation in the motor system leads to impaired movement. Curr Opin Neurobiol. (2007) 17:65664. doi: 10.1016/j.conb.2007.12.001

27. D’Andrea A, Chella F, Marshall TR, Pizzella V, Romani GL, Jensen O, et al. Alpha and alpha-beta phase synchronization mediate the recruitment of the visuospatial attention network through the Superior Longitudinal Fasciculus. NeuroImage. (2019) 188:722-32. doi: 10.1016/j.neuroimage.2018.12.056

28. Zareian B, Maboudi K, Daliri MR, Abrishami Moghaddam H, Treue S, Esghaei M. et al. Attention strengthens across-trial pre-stimulus phase coherence in visual cortex, enhancing stimulus processing. Sci Rep. (2020) 10:4837. doi: 10.1038/s41598-020-61359-7

29. Ara A, Marco-Pallarés J. Fronto-temporal theta phase-synchronization underlies music-evoked pleasantness. Neuroimage. (2020) 212:116665. doi: 10.1016/j.neuroimage.2020.116665

30. Ahn S, Cho H, Kwon M, Kim K, Kwon H, Kim BS, et al. Interbrain phase synchronization during turn-taking verbal interaction-a hyperscanning study using simultaneous EEG/MEG. Hum Brain Mapp. (2018) 39:17188. doi: 10.1002/hbm.23834

31. Bob P, Palus M, Susta M, Glaslova K. EEG phase synchronization in patients with paranoid schizophrenia. Neurosci Lett. (2008) 447:737. doi: 10.1016/j.neulet.2008.09.055

32. Pusil S, Dimitriadis SI, López ME, Pereda E, Maestú F. Aberrant MEG multi-frequency phase temporal synchronization predicts conversion from mild cognitive impairment-to-Alzheimer's disease. NeuroImage. (2019) 24:101972. doi: 10.1016/j.nicl.2019.101972

33. Nenadovic V, Perez Velazquez JL, Hutchison JS. Phase synchronization in electroencephalographic recordings prognosticates outcome in paediatric coma. PLoS ONE. (2014) 9:e94942. doi: 10.1371/journal.pone.0094942

34. Miocinovic S, de Hemptinne C, Qasim S, Ostrem JL, Starr PA. Patterns of cortical synchronization in isolated dystonia compared with Parkinson disease. JAMA Neurol. (2015) 72:1244-51. doi: 10.1001/jamaneurol.2015.2561

35. Lee S, Liu A, Wang ZJ, McKeown MJ. Abnormal phase coupling in Parkinson's disease and normalization effects of subthreshold vestibular stimulation. Front Hum Neurosc. (2019) 13:118. doi: 10.3389/fnhum.2019.00118

36. Ruiz MH, Senghaas P, Grossbach M, Jabusch HC, Bangert M, Hummel $\mathrm{F}$, et al. Defective inhibition and inter-regional phase synchronization in pianists with musician's dystonia: an EEG study. Hum Brain Mapp. (2009) 30:2689-700. doi: 10.1002/hbm.20700

37. Delussi M, Nazzaro V, Ricci K, de Tommaso M. EEG functional connectivity and cognitive variables in premanifest and manifest Huntington's disease: EEG Low-Resolution Brain Electromagnetic Tomography (LORETA) Study. Front Physiol. (2020) 11:612325. doi: 10.3389/fphys.2020.612325

38. Pfurtscheller G, Lopes da Silva FH. Event-related EEG/MEG synchronization and desynchronization: basic principles. Clin Neurophysiol. (1999) 110:184257. doi: 10.1016/S1388-2457(99)00141-8

39. Beuter A, Modolo J. Delayed and lasting effects of deep brain stimulation on locomotion in Parkinson's disease. Chaos. (2009) 19:026114. doi: 10.1063/1.3127585

40. Weerasinghe G, Duchet B, Cagnan H, Brown P, Bick C, Bogacz R. Predicting the effects of deep brain stimulation using a reduced coupled oscillator model. PLoS Comput Biol. (2019) 15:e1006575. doi: 10.1371/journal.pcbi.1006575
41. Lachaux JP, Rodriguez E, Martinerie J, Varela FJ. Measuring phase synchrony in brain signals. Hum Brain Mapp. (1999) 8:194-208. doi: 10.1002/(SICI)1097-0193(1999)8:4\&lt;194::AID-HBM4\& gt;3.0.CO;2-C

42. Noda Y, Zomorrodi R, Vila-Rodriguez F, Downar J, Farzan F, Cash RFH, et al. Impaired neuroplasticity in the prefrontal cortex in depression indexed through paired associative stimulation. Depress Anxiety. (2018) 35:44856. doi: $10.1002 /$ da. 22738

43. Voytovych H, Kriváneková L, Ziemann U. Lithium: a switch from LTDto LTP-like plasticity in human cortex. Neuropharmacology. (2012) 63:2749. doi: 10.1016/j.neuropharm.2012.03.023

44. Suppa A, Marsili L, Belvisi D, Conte A, Iezzi E, Modugno N, et al. Lack of LTP-like plasticity in primary motor cortex in Parkinson's disease. Exp Neurol. (2011) 227:296-301. doi: 10.1016/j.expneurol.2010.11.020

45. Tisch S, Limousin P. Neurophysiological insights in dystonia and its response to deep brain stimulation treatment. Exp Brain Res. (2020) 238:164557. doi: 10.1007/s00221-020-05833-8

46. Brown P, Eusebio A. Paradoxes of functional neurosurgery: clues from basal ganglia recordings. Mov Disord. (2008) 23:12-20. doi: 10.1002/mds.21796

47. Krack P, Batir A, Van Blercom N, Chabardes S, Fraix V, Ardouin C, et al. Five year follow-up of bilateral stimulation of the subthalamic nucleus in advanced Parkinson's disease. N Engl J Med. (2003) 349:192534. doi: 10.1056/NEJMoa035275

48. Udupa K, Chen R. Motor cortical plasticity in Parkinson's disease. Front Neurol. (2013) 4:128. doi: 10.3389/fneur.2013.00128

49. Quartarone A, Ruge D. How many types of dystonia? pathophysiological considerations. Front Neurol. (2018) 9:12. doi: 10.3389/fneur.2018.00012

50. Suppa A, Marsili L, Di Stasio F, Berardelli I, Roselli V, Pasquini M, et al. Cortical and brainstem plasticity in Tourette syndrome and obsessivecompulsive disorder. Mov Disord. (2014) 29:1523-31. doi: 10.1002/mds. 25960

51. Nerrant E, Gonzalez V, Milesi C, Vasques X, Ruge D, Roujeau T, et al. Deep brain stimulation treated dystonia-trajectory via status dystonicus. Mov Disord. (2018) 33:1168-73. doi: 10.1002/mds.27357

52. Wiethoff S, Hamada M, Rothwell JC. Variability in response to transcranial direct current stimulation of the motor cortex. Brain Stimul. (2014) 7:46875. doi: 10.1016/j.brs.2014.02.003

53. Pedroarena-Leal N, Heidemeyer L, Trenado C, Ruge D. Human depotentiation following induction of spike timing dependent plasticity. Biomedicines. (2018) 6:71. doi: 10.3390/biomedicines6020071

54. Honkanen EA, Korpela J, Pekkonen E, Kaasinen V, Reich MM, Joutsa J. Reappearance of symptoms after GPi-DBS discontinuation in cervical dystonia. Mov Disord. (2021) 8:406-11. doi: 10.1002/mdc3.13162

Conflict of Interest: The authors declare that the research was conducted in the absence of any commercial or financial relationships that could be construed as a potential conflict of interest.

Publisher's Note: All claims expressed in this article are solely those of the authors and do not necessarily represent those of their affiliated organizations, or those of the publisher, the editors and the reviewers. Any product that may be evaluated in this article, or claim that may be made by its manufacturer, is not guaranteed or endorsed by the publisher.

Copyright (C) 2021 Trenado, Cif, Pedroarena-Leal and Ruge. This is an open-access article distributed under the terms of the Creative Commons Attribution License (CC $B Y)$. The use, distribution or reproduction in other forums is permitted, provided the original author(s) and the copyright owner(s) are credited and that the original publication in this journal is cited, in accordance with accepted academic practice. No use, distribution or reproduction is permitted which does not comply with these terms. 